\title{
MAXIMAL REPRESENTATION DIMENSION OF FINITE $p$-GROUPS
}

\author{
SHANE CERNELE, MASOUD KAMGARPOUR, AND ZINOVY REICHSTEIN
}

\begin{abstract}
The representation dimension $\operatorname{rdim}(G)$ of a finite group $G$ is the smallest positive integer $m$ for which there exists an embedding of $G$ in $\mathrm{GL}_{m}(\mathbb{C})$. In this paper we find the largest value of $\operatorname{rdim}(G)$, as $G$ ranges over all groups of order $p^{n}$, for a fixed prime $p$ and a fixed exponent $n \geq 1$.
\end{abstract}

\section{INTRODUCTION}

The representation dimension of a finite group $G$, denoted by $\operatorname{rdim}(G)$, is the minimal dimension of a faithful complex linear representation of $G$. In this paper we determine the maximal representation dimension of a group of order $p^{n}$. We are motivated by a recent result of N. Karpenko and A. Merkurjev [KM07, Theorem 4.1], which states that if $G$ is a finite $p$-group then the essential dimension of $G$ is equal to $\operatorname{rdim}(G)$. For a detailed discussion of the notion of essential dimension for finite groups (which will not be used in this paper), see BR97 or JLY02, §8]. We also note that a related invariant, the minimal dimension of a faithful complex projective representation of $G$, has been extensively studied for finite simple groups $G$; for an overview, see [TZ00, $\S 3]$.

Let $G$ be a $p$-group of order $p^{n}$ and $r$ be the rank of the centre $Z(G)$. A representation of $G$ is faithful if and only if its restriction to $Z(G)$ is faithful. Using this fact it is easy to see that a faithful representation $\rho$ of $G$ of minimal dimension decomposes as a direct sum

$$
\rho=\rho_{1} \oplus \cdots \oplus \rho_{r}
$$

of exactly $r$ irreducibles; cf. MR09, Theorem 1.2]. Since the dimension of any irreducible representation of $G$ is $\leq \sqrt{[G: Z(G)]}$ (see, e.g., W03, Corollary 3.11]) and $|Z(G)| \geq p^{r}$, we conclude that

$$
\operatorname{rdim}(G) \leq r p^{\lfloor(n-r) / 2\rfloor} .
$$

2000 Mathematics Subject Classification. 20C15, 20 D15.

Key words and phrases. p-group, representation dimension, symplectic subspace, generalized Heisenberg group.

The authors gratefully acknowledge financial support by the Natural Sciences and Engineering Research Council of Canada. 
Let

$$
f_{p}(n):=\max _{r \in \mathbb{N}}\left(r p^{\lfloor(n-r) / 2\rfloor}\right) .
$$

It is easy to check that $f_{p}(n)$ is given by the following table:

\begin{tabular}{|c|c|c|}
\hline$n$ & $p$ & $f_{p}(n)$ \\
\hline even & arbitrary & $2 p^{(n-2) / 2}$ \\
\hline odd & odd & $p^{(n-1) / 2}$ \\
\hline odd, $\geq 3$ & 2 & $3 p^{(n-3) / 2}$ \\
\hline 1 & 2 & 1 \\
\hline
\end{tabular}

We are now ready to state the main result of this paper.

Theorem 1. Let $p$ be a prime and $n$ be a positive integer. For almost all pairs $(p, n)$, the maximal value of $\operatorname{rdim}(G)$, as $G$ ranges over all groups of order $p^{n}$, equals $f_{p}(n)$. The exceptional cases are

$$
(p, n)=(2,5),(2,7) \text { and }(p, 4), \text { where } p \text { is odd. }
$$

In these cases the maximal representation dimension is 5, 10, and $p+1$, respectively.

The proof will show that the maximal value of $\operatorname{rdim}(G)$, as $G$ ranges over all groups of order $p^{n}$, is always attained for a group $G$ of nilpotency class $\leq 2$. Moreover, if $(p, n)$ is non-exceptional, $n \geq 3$ and $(p, n) \neq(2,3),(2,4)$, the maximum is attained on a special class of $p$-groups of nilpotency class 2. We call these groups generalized Heisenberg groups since their representation theory looks very similar to the usual Heisenberg group (the group of unipotent upper triangular $3 \times 3$ matrices); see Section 2.4

The rest of this paper is structured as follows. In $\$ 2$ we introduce generalized Heisenberg groups and study their irreducible representations. In 93 , we prove Theorem 1 .

Acknowledgement. We would like to thank Hannah Cairns, Robert Guralnick, Chris Parker, Burt Totaro, and Robert Wilson for helpful discussions. We are also grateful to the referee for constructive comments.

\section{Generalized Heisenberg groups}

2.1. Spaces of alternating forms. Let $V$ be a finite dimensional vector space over an arbitrary field $F$. Let $\mathcal{A}(V)$ denote the space of bilinear alternating forms on $V$; that is, linear maps $b: V \otimes V \rightarrow F$ satisfying $b(v, v)=0$.

Let $K$ be a subspace of $\mathcal{A}(V)$. Then $K$ defines a map $\omega_{K}: V \times V \rightarrow K^{*}$ as follows. Let $j: \mathcal{A}(V)^{*} \rightarrow K^{*}$ denote the dual of the natural injection $K \hookrightarrow \mathcal{A}(V)$. Then $\omega_{K}$ is defined to be the composition

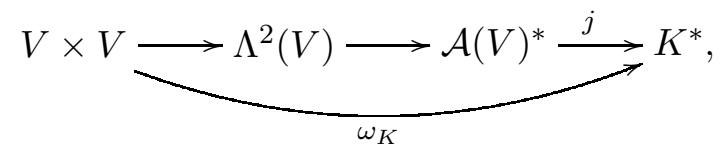


where the first map is the natural projection and the second one is the canonical identification of the two spaces.

\subsection{Symplectic subspaces.}

Definition 2. A subspace $K \subseteq \mathcal{A}(V)$ is symplectic if every nonzero element of $K$ is non-degenerate, as a bilinear form on $V$.

Remark 3. Equivalently, $K \subset \mathcal{A}(V)$ is symplectic if and only if for every nonzero linear map $K^{*} \rightarrow F$ the composition $V \times V \stackrel{\omega_{K}}{\longrightarrow} K^{*} \rightarrow F$ is nondegenerate.

Clearly nontrivial symplectic subspaces of $\mathcal{A}(V)$ can exist only if $\operatorname{dim}(V)$ is even.

Lemma 4. Suppose $V$ is an $F$-vector space of dimension $2 m$. If $F$ admits a field extension of degree $m$ then there exists an $m$-dimensional symplectic subspace $K \subset \mathcal{A}$.

Proof. Choosing a basis of $V$, we can identify $\mathcal{A}(V)$ with the space of alternating $2 m \times 2 m$-matrices. Let $f: \mathrm{M}_{m}(F) \rightarrow \mathcal{A}(V)$ be the linear map

$$
A \mapsto\left[\begin{array}{cc}
0 & A \\
-A^{T} & 0
\end{array}\right]
$$

If $W$ is a linear subspace of $\mathrm{M}_{m}(F)=\operatorname{End}_{F}\left(F^{m}\right)$ such that $W \backslash\{0\} \subset$ $\mathrm{GL}_{m}(F)$ then $K=f(W)$ is a symplectic subspace.

It thus remains to construct an $m$-dimensional linear subspace $W$ of $\mathrm{M}_{m}(F)$ such that $W \backslash\{0\} \subset \mathrm{GL}_{m}(F)$. Let $E$ be a degree $m$ field extension of $F$. Then $E$ acts on itself by left multiplication. This gives an $F$-vector space embedding of $\Psi: E \hookrightarrow \operatorname{End}_{F}(E)$ such that $\Psi(e)$ is invertible for all $e \neq 0$.

2.3. Groups associated to spaces of alternating forms. Let $V$ be a finite-dimensional vector space over a field $F$. Let $K$ be a subspace of $\mathcal{A}(V)$ and let $\omega_{K}$ denote the induced map $V \times V \rightarrow K^{*}$, see (3). Choose a bilinear map $\beta: V \times V \rightarrow K^{*}$ such that

$$
\omega_{K}(v, w)=\beta(v, w)-\beta(w, v) .
$$

To see that this can always be done, note that if $\left\{e_{i}\right\}$ is a basis of $V$, we can define $\beta$ by

$$
\beta\left(e_{i}, e_{j}\right)=\left\{\begin{array}{l}
\omega_{K}\left(e_{i}, e_{j}\right), \text { if } i>j \text { and } \\
0, \text { otherwise. }
\end{array}\right.
$$

We also remark that $\beta$ is uniquely determined by $\omega_{K}$, up to adding a symmetric bilinear form $V \times V \rightarrow K^{*}$.

Definition 5. Let $H=H(V, K, \beta)$ denote the group whose underlying set is $V \times K^{*}$ and whose multiplication is given by

$$
(v, t) \cdot\left(v^{\prime}, t^{\prime}\right)=\left(v+v^{\prime}, t+t^{\prime}+\beta\left(v, v^{\prime}\right)\right) .
$$


If $K$ is a symplectic subspace, we will refer to $H$ as a generalized Heisenberg group.

Example 6. Suppose $\omega$ is a nondegenerate alternating bilinear form on $V=F \oplus F$, where $F$ is a field of characteristic not equal to 2 . Let $K$ be the span of $\omega$ in $A(V)$. Then $H\left(V, K, \frac{1}{2} \omega\right)$ is isomorphic to the group of unipotent upper triangular $3 \times 3$ matrices over $F$. This group is known as the Heisenberg group.

Remark 7. It is easy to see that (5) is indeed a group law with the inverse given by $(v, t)^{-1}=(-v,-t+\beta(v, v))$ and the commutator given by

$$
\left[\left(v_{1}, t_{1}\right),\left(v_{2}, t_{2}\right)\right]=\left(0, \omega_{K}\left(v_{1}, v_{2}\right)\right) .
$$

As $\omega_{K}$ is surjective, we see that $[H, H]=K^{*}$. Moreover, (6) also shows that $K^{*} \subset Z(H)$, and that equality holds unless the intersection $\cap_{k \in K} \operatorname{ker}(k)$ is nontrivial. In particular, $Z(H)=K^{*}$ if $K$ contains a symplectic form.

Remark 8. A non-abelian finite $p$-group $S$ is called special if $Z(S)=[S, S]$ and $S /[S, S]$ is elementary abelian; see [HH56, §2.3]. Suppose K is a subspace of $\mathcal{A}(V)$ such that $\cap_{k \in K} \operatorname{ker}(k)$ is trivial. Then over the finite field $\mathbb{F}_{p}$, the groups $H(V, K, \beta)$ are examples of non-abelian special $p$-groups. We are grateful to the referee for pointing this out.

Remark 9. If $\beta$ and $\beta^{\prime}$ both satisfy (4) then $H(V, K, \beta)$ may not be isomorphic to $H\left(V, K, \beta^{\prime}\right)$. For example, let $V$ be a 2 -dimensional vector space over $F=\mathbb{F}_{2}, K$ be the one-dimensional (symplectic) subspace generated by $\left[\begin{array}{ll}0 & 1 \\ 1 & 0\end{array}\right]$, and $\beta, \beta^{\prime}$ be bilinear forms on $V$ defined by $\left[\begin{array}{ll}1 & 1 \\ 0 & 1\end{array}\right]$ and $\left[\begin{array}{ll}0 & 1 \\ 0 & 0\end{array}\right]$, respectively. Then $\beta$ and $\beta^{\prime}$ both satisfy (4), but $H(V, K, \beta)$ is isomorphic to the quaternion group while $H\left(V, K, \beta^{\prime}\right)$ is isomorphic to the dihedral group of order 8 .

On the other hand, it is easy to see that $H(V, K, \beta)$ and $H\left(V, K, \beta^{\prime}\right)$ are always isoclinic. (Two groups $S$ and $T$ are isoclinic if there are isomorphisms $f: S / Z(S) \rightarrow T / Z(T)$ and $g:[S, S] \rightarrow[T, T]$ such that if $a, b \in S$ and $a^{\prime}, b^{\prime} \in T$ with $f(a Z(S))=a^{\prime} Z(T)$ and $f(b Z(S))=b^{\prime} Z(T)$, then we have $g([a, b])=\left[a^{\prime}, b^{\prime}\right]$, see [H40].)

2.4. Representations. Let $p$ be an arbitrary prime and let $F=\mathbb{F}_{p}$ be the finite field of $p$ elements. Fix, once and for all, a homomorphism $\tau$ : $\left(\mathbb{F}_{p},+\right) \hookrightarrow \mathbb{C}^{*}$. Let $W$ be a vector space over $F$. Using $\tau$, we identify the algebraic dual $W^{*}=\operatorname{Hom}(W, F)$ with the Pontriyagin dual $\operatorname{Hom}\left(W, \mathbb{C}^{*}\right)$. It is clear that a bilinear alternating map $W \times W \rightarrow \mathbb{F}_{p}$ is non-degenerate if and only if the composition $W \times W \rightarrow \mathbb{F}_{p} \stackrel{\tau}{\longrightarrow} \mathbb{C}^{\times}$is non-degenerate.

Now let $V$ be a vector space over $F, K$ a subspace of $\mathcal{A}(V)$, and $\omega=\omega_{K}$ the associated map. Choose $\beta$ satisfying (4) and let $G=H(V, K, \beta)=$ $V \times K^{*}$. Recall that $K^{*}$ is in the center of $G$ (Remark 7); in particular, it acts via a character on every irreducible representation of $G$. 
Lemma 10. Let $\rho$ be an irreducible representation of $G$ such that $K^{*}$ acts by $\psi$. Assume $\psi \circ \omega: V \times V \rightarrow \mathbb{C}^{\times}$is non-degenerate.

(a) If $g \in G, g \notin K^{*}$, then $\operatorname{Tr}(\rho(g))=0$.

(b) $\operatorname{dim}(\rho)=\sqrt{|V|}$.

(c) $\rho$ is uniquely determined (up to isomorphism) by $\psi$.

Proof. (a) Let $g \in G \backslash K^{*}$. Since $\psi \circ \omega$ is non-degenerate there exists $h \in G$ such that $\psi \circ \omega\left(g K^{*}, h K^{*}\right) \neq 1$. Observe that $\rho([g, h])=\psi([g, h]) \mathrm{Id}$, and that $\rho\left(h^{-1} g h\right)=\rho(g) \rho([g, h])$. Taking the trace of both sides, we have $\operatorname{Tr}(\rho(g))=\psi([g, h]) \operatorname{Tr}(\rho(g))$. Since $\psi([g, h]) \neq 1$ we must have $\operatorname{Tr}(\rho(g))=0$.

(b) Since $\rho$ is irreducible, and the trace of $\rho$ vanishes outside of $K^{*}$, we have:

$$
\begin{aligned}
1 & =\frac{1}{|G|} \sum_{g \in G} \operatorname{Tr}(\rho(g)) \overline{\operatorname{Tr}(\rho(g))} \\
& =\frac{1}{|G|} \sum_{g \in K^{*}} \operatorname{Tr}(\rho(g)) \overline{\operatorname{Tr}(\rho(g))} \\
& =\frac{1}{|G|} \operatorname{dim}(\rho)^{2} \sum_{g \in K^{*}} \operatorname{Tr}(\psi(g)) \overline{\operatorname{Tr}(\psi(g))} \\
& =\operatorname{dim}(\rho)^{2} \frac{\left|K^{*}\right|}{|G|}
\end{aligned}
$$

Thus $\operatorname{dim} \rho=\sqrt{|G| /\left|K^{*}\right|}=\sqrt{|V|}$.

(c) We have completely described the character of $\rho$, and it follows that $\rho$ is uniquely determined by $\psi$. Indeed,

$$
\operatorname{Tr}(\rho(g))= \begin{cases}\sqrt{|V|} \cdot \psi(g), & \text { if } g \in K^{*} \text { and } \\ 0 & \text { otherwise. }\end{cases}
$$

In view of Remark 3, the following proposition is a direct consequence of the above lemma.

Proposition 11. The irreducible representations of a generalized Heisenberg group $H=H(V, K, \beta)$ are exhausted by the following list:

(i) $|V|$ one-dimensional representations, one for every character of $V$.

(ii) $|K|-1$ representations of dimension $\sqrt{|V|}$, one for every nontrivial character $\psi: K^{*} \rightarrow \mathbb{C}^{\times}$.

The next corollary is also immediate upon observing the centre of a generalized Heisenberg groups $H=H(G, K, \beta)$ equals $K^{*}$; see Remark 7 .

Corollary 12. The representation dimension of a generalized Heisenberg group $H=H(V, K, \beta)$ equals $\operatorname{dim}(K) \sqrt{|V|}$. 
If $G$ is a finite Heisenberg group in the usual sense (as in Example 6) then for each nontrivial character $\chi$ of $Z(G)$ there is a unique irreducible representation $\psi$ of $G$ whose central character is $\chi$; cf. [GH07, §1.1]. This is a finite group variant of the celebrated Stone-von Neumann Theorem. For a detailed discussion of the history and the various forms of the Stone-von Neumann theorem we refer the reader to R04. We conclude this section with another immediate corollary of Proposition [11 which tells us that over the field $\mathbb{F}_{p}$ every generalized Heisenberg group has the Stone-von Neumann property. This corollary will not be needed in the sequel.

Corollary 13. Two irreducible representations of a generalized Heisenberg group with the same nontrivial central character are isomorphic.

Corollary 13 is the reason we chose to use the term "generalized Heisenberg group" in reference to the groups $H(V, K, \beta)$, where $\mathrm{K}$ is a symplectic subspace. Special $p$-groups (Remark 8) which are not generalized Heisenberg groups may not have the Stone-von Neumann property; see Remark 18.

\section{Proof of Theorem 1}

The case where $n \leq 2$ is trivial; clearly $\operatorname{rdim}(G)=\operatorname{rank}(G)$ if $G$ is abelian. We will thus assume that $n \geq 3$.

In the non-exceptional cases of the theorem, in view of the inequality (2), it suffices to construct a group $G$ of order $p^{n}$ with $\operatorname{rdim}(G)=f_{p}(n)$. Here $f_{p}(n)$ is the function defined just before the statement of Theorem 1 .

If $(p, n)=(2,3)$ or $(2,4)$, we take $G$ to be the elementary abelian group $(\mathbb{Z} / 2 \mathbb{Z})^{3}$ and $(\mathbb{Z} / 2 \mathbb{Z})^{4}$, yielding the desired representation dimension of 3 and 4 , respectively. For all other non-exceptional pairs $(p, n)$, we take $G$ to be a generalized Heisenberg group as described in the table below. Here $H(V, K)$ stands for $H(V, K, \beta)$, for some $\beta$ as in (4). In each instance, the existence of a symplectic subspace $K$ of suitable dimension is guaranteed by Lemma 4 and the value of $\operatorname{rdim}(H(V, K))$ is given by Corollary 12 .

\begin{tabular}{|c|c|c|c|c|}
\hline$n$ & $p$ & $\operatorname{dim}(V)$ & $\operatorname{dim}(K)$ & $\operatorname{rdim}(H(V, K))$ \\
\hline even, $\geq 6$ & arbitrary & $n-2$ & 2 & $2 p^{(n-2) / 2}$ \\
\hline odd, $\geq 3$ & odd & $n-1$ & 1 & $p^{(n-1) / 2}$ \\
\hline odd, $\geq 9$ & 2 & $n-3$ & 3 & $3 p^{(n-3) / 2}$ \\
\hline
\end{tabular}

This settles the generic case of Theorem 1, We now turn our attention to the exceptional cases. We will need the following upper bound on $\operatorname{rdim}(G)$, strengthening (2).

Let $\Omega_{1}(Z(G))$ be the subgroup of elements $g \in Z(G)$ such that $g^{p}=1$.

Lemma 14. Let $G$ be a p-group and $r=\operatorname{rank}(Z(G))=\operatorname{rank}\left(\Omega_{1}(Z(G))\right)$.

(a) Let $\rho_{1}$ be an irreducible representation of $G$ such that $\operatorname{Ker}\left(\rho_{1}\right)$ does not contain $\Omega_{1}(Z(G))$. Then there are irreducible representations $\rho_{2}, \ldots, \rho_{r}$ 
of $G$ such that $\rho_{1} \oplus \cdots \oplus \rho_{r}$ is faithful. In particular,

$$
\operatorname{rdim}(G) \leq \operatorname{dim}\left(\rho_{1}\right)+(r-1) \sqrt{[G: Z(G)]} .
$$

(b) If $\Omega_{1}(Z(G))$ is not contained in $[G, G]$, then

$$
\operatorname{rdim}(G) \leq 1+(r-1) \sqrt{[G: Z(G)]} .
$$

The lemma can be deduced from [KM07, Remark 4.7] or [MR09, Theorem 1.2]; for the sake of completeness we give a self-contained proof.

Proof. (a) Let $\chi_{1}$ be the restriction to $\Omega_{1}(Z(G))$ of the central character of $\rho_{1}$. By our assumption $\chi_{1}$ is nontrivial. Complete $\chi_{1}$ to a basis $\chi_{1}, \chi_{2}, \ldots, \chi_{r}$ of the $r$-dimensional $\mathbb{F}_{p}$-vector space $\Omega_{1}(Z(G))^{*}$ and choose an irreducible representation $\rho_{i}$ such that $\Omega_{1}\left(Z(G)\right.$ ) acts by $\chi_{i}$. (The representation $\rho_{i}$ can be taken to be any irreducible component of the induced representation $\operatorname{Ind}_{\Omega_{1}(Z(G))}^{G}(\chi)$.) The restriction of $\rho:=\rho_{1} \oplus \cdots \oplus \rho_{r}$ to $\Omega_{1}(Z(G))$ is faithful. Hence, $\rho$ is a faithful representation of $G$. As we mentioned in the introduction $\operatorname{dim}\left(\rho_{i}\right) \leq \sqrt{[G: Z(G)]}$ for every $i \geq 2$, and part (a) follows.

(b) By our assumption there exists a one-dimensional representation $\rho_{1}$ of $G$ whose restriction to $\Omega_{1}(Z(G))$ is nontrivial. Now apply part (a).

We are now ready to prove Theorem 1 in the three exceptional cases.

\subsection{Exceptional case 1: $p$ is odd and $n=4$.}

Lemma 15. Let $p$ be an odd prime and $G$ be a group of order $p^{4}$.

(a) Then $\operatorname{rdim}(G) \leq p+1$.

(b) Suppose $Z(G) \simeq(\mathbb{Z} / p \mathbb{Z})^{2}$ and $G / Z(G) \simeq(\mathbb{Z} / p \mathbb{Z})^{2}$. Then $\operatorname{rdim}(G)=$ $p+1$.

Proof. (a) We argue by contradiction. Assume there exists a group of order $p^{4}$ such that $\operatorname{rdim}(G) \geq p+2$. If $|Z(G)| \geq p^{3}$ or $G / Z(G)$ is cyclic then $G$ is abelian and $\operatorname{rdim}(G)=\operatorname{rank}(G) \leq 4 \leq p+1$, a contradiction. If $Z(G)$ is cyclic then $\operatorname{rdim}(G) \leq p$ by (2), again a contradiction.

Thus $Z(G) \simeq G / Z(G) \simeq(\mathbb{Z} / p \mathbb{Z})^{2}$. This reduces part (a) to part (b).

(b) Here $\Omega_{1}(Z(G))=Z(G)$ has rank 2. Hence, a faithful representation $\rho$ of $G$ of minimal dimension is the sum of two irreducibles $\rho_{1} \oplus \rho_{2}$, as in (1), each of dimension 1 or $p$.

Clearly $\operatorname{dim}\left(\rho_{1}\right)=\operatorname{dim}\left(\rho_{2}\right)=1$ is not possible, since in this case $G$ would be abelian, contradicting $[G: Z(G)]=p^{2}$. It thus remains to show that $\operatorname{rdim}(G) \leq p+1$. Since $G / Z(G)$ is abelian, $[G, G] \subset Z(G)$. Hence, by Lemma 14(b) we only need to establish that $[G, G] \subsetneq Z(G)$.

To show that $[G, G] \subsetneq Z(G)$, note that the commutator map

$$
\begin{aligned}
\Psi: G / Z(G) \times G / Z(G) & \rightarrow[G, G] \\
\left(g Z(G), g^{\prime} Z(G)\right) & \mapsto\left[g, g^{\prime}\right]
\end{aligned}
$$

can be thought of as an alternating bilinear map from $\mathbb{F}_{p}^{2}$ to itself. Viewed in this way, $\Psi$ can be written as $\Psi\left(v, v^{\prime}\right)=\left(w_{1}\left(v, v^{\prime}\right), w_{2}\left(v, v^{\prime}\right)\right)$ for alternating 
maps $w_{1}$ and $w_{2}$ from $\left(\mathbb{F}_{p}\right)^{2}$ to $\mathbb{F}_{p}$. Since the space of alternating maps is a one-dimensional vector space over $\mathbb{F}_{p}, w_{1}$ and $w_{2}$ are scalar multiples of each other. Hence, the image of $\Psi$ is a cyclic group of order $p$, and $[G, G] \subsetneq Z(G)$, as claimed.

To finish the proof of Theorem 1 in this case, note that $G=\mathbb{Z} / p \mathbb{Z} \times G_{0}$, where $G_{0}$ is a non-abelian group of order $p^{3}$, satisfies the conditions of Lemma 15(b). Thus the maximal representation dimension of a group of order $p^{4}$ is $p+1$, for any odd prime $p$.

\subsection{Exceptional case 2: $p=2$ and $n=5$.}

Lemma 16. Let $G$ be a group of order 32 . Then $\operatorname{rdim}(G) \leq 5$.

Proof. We argue by contradiction. Assume there exists a group of order 32 and representation dimension $\geq 6$. Let $r=\operatorname{rank}(Z(G))$. Then $1 \leq r \leq 5$ and (2) shows that $\operatorname{rdim}(G) \leq 5$ for every $r \neq 3$.

Thus we may assume $r=3$. If $|Z(G)| \geq 16$ or $G / Z(G)$ is cyclic then $G$ is abelian, and $\operatorname{rdim}(G)=\operatorname{rank}(G) \leq 5$. We conclude that $Z(G) \simeq(\mathbb{Z} / 2 \mathbb{Z})^{3}$ and $G / Z(G) \simeq(\mathbb{Z} / 2 \mathbb{Z})^{2}$. Applying the same argument as in the proof of Lemma 15 (b), we see that $[G, G] \subsetneq Z(G)$, and hence $\operatorname{rdim}(G) \leq 5$ by Lemma 14(b), a contradiction.

To finish the proof of Theorem 1 in this case, note that the elementary abelian group of order $2^{5}$ has representation dimension 5 . Thus the maximal representation dimension of a group of order $2^{5}$ is 5 .

\subsection{Exceptional case 3: $p=2$ and $n=7$.}

Lemma 17. If $|G|=128$ then $\operatorname{rdim}(G) \leq 10$.

Proof. Again, we argue by contradiction. Assume there exists a group $G$ of order 128 and representation dimension $\geq 11$. Let $r$ be the rank of $Z(G)$. By (2),$r=3$; otherwise we would have $\operatorname{rdim}(G) \leq 10$.

As we explained in the introduction, this implies that a faithful representation $\rho$ of $G$ of minimal dimension is the direct sum of three irreducibles $\rho_{1}$, $\rho_{2}$ and $\rho_{3}$, each of dimension $\leq \sqrt{2^{7} /|Z(G)|}$. If $|Z(G)|>8$, then $\operatorname{dim}\left(\rho_{i}\right) \leq 2$ and $\operatorname{rdim}(G)=\operatorname{dim}\left(\rho_{1}\right)+\operatorname{dim}\left(\rho_{2}\right)+\operatorname{dim}\left(\rho_{3}\right) \leq 6$, a contradiction.

Therefore, $Z(G) \cong(\mathbb{Z} / 2 \mathbb{Z})^{3}$ and $\operatorname{dim}\left(\rho_{1}\right)=\operatorname{dim}\left(\rho_{2}\right)=\operatorname{dim}\left(\rho_{3}\right)=4$. By Lemma 14(a) this implies that the kernel of every irreducible representation of $G$ of dimension 1 or 2 must contain $Z(G)$. In other words, any such representation factors through the group $G / Z(G)$ of order 16. Consequently, if $m_{i}$ is the number of irreducible representations of $G$ of dimension $i$ then $m_{1}+4 m_{2}=16$. We can now appeal to [JNO90, Tables I and II], to show that no group of order $2^{7}$ has these properties. From Table I we can determine which groups $G$ (up to isoclinism, cf. Remark 9) have $|Z(G)|=8$ and using Table II we can determine $m_{1}$ and $m_{2}$ for these groups. There is no group $G$ with $|Z(G)|=8$ and $m_{1}+4 m_{2}=16$. 
We will now construct an example of a group $G$ of order $2^{7}$ with $\operatorname{rdim}(G)=$ 10. Let $V=\left(\mathbb{F}_{2}\right)^{4}$ and let $K$ be the 3-dimensional subspace of $A(V)$ generated by the following three elements:

$$
\left[\begin{array}{llll}
0 & 0 & 0 & 1 \\
0 & 0 & 1 & 0 \\
0 & 1 & 0 & 0 \\
1 & 0 & 0 & 0
\end{array}\right], \quad\left[\begin{array}{llll}
0 & 0 & 1 & 0 \\
0 & 0 & 1 & 1 \\
1 & 1 & 0 & 0 \\
0 & 1 & 0 & 0
\end{array}\right], \quad\left[\begin{array}{llll}
0 & 0 & 1 & 1 \\
0 & 0 & 0 & 1 \\
1 & 0 & 0 & 1 \\
1 & 1 & 1 & 0
\end{array}\right] .
$$

Let $G:=H(V, K, \beta)=V \times K^{*}$ for some $\beta$ as in (44). Note that $K$ contains only one non-zero degenerate element (the sum of the three generators). In other words, there is only one non-trivial character $\chi$ of $K^{*}$ such that $\chi \circ \omega: V \times V \rightarrow \mathbb{C}^{\times}$is degenerate. By Remark 7

$$
[G, G]=Z(G)=K^{*} .
$$

Let $\rho$ be a faithful representation of $G$ of minimal dimension. As we explained in the Introduction, $\rho$ is the sum of $\operatorname{rank}(Z(G))=3$ irreducibles. Denote them by $\rho_{1}, \rho_{2}$, and $\rho_{3}$, and their central characters by $\chi_{1}, \chi_{2}$ and $\chi_{3}$, respectively. Since $\rho$ is faithful, $\chi_{1}, \chi_{2}$ and $\chi_{3}$ form an $\mathbb{F}_{2}$-basis of $\Omega_{1}(Z(G))^{*} \simeq(\mathbb{Z} / 2 \mathbb{Z})^{3}$. By Lemma 10 , for each nontrivial character $\chi$ of $K^{*}$ except one, there is a unique irreducible representation $\psi$ of $G$ such that $\chi$ is the central character to $\psi$, and $\operatorname{dim} \psi=4$. Thus at least 2 of the irreducible components of $\rho$, say, $\rho_{1}$ and $\rho_{2}$ must have dimension 4. By Lemma 17. $\operatorname{dim}(\rho) \leq 10$, i.e., $\operatorname{dim}\left(\rho_{3}\right) \leq 2$. But every one-dimensional representation of $G$ has trivial central character. We conclude that $\operatorname{dim}\left(\rho_{3}\right)=2$ and consequently $\operatorname{rdim}(G)=\operatorname{dim}(\rho)=4+4+2=10$.

Thus the maximal representation dimension of a group of order $2^{7}$ is 10 .

Remark 18. The group $G$ constructed above has 16 one-dimensional representations with trivial central character, 4 two-dimensional representations with non-trivial degenerate central character, and 6 four-dimensional representations with pair-wise distinct non-degenerate central characters. In view of (7), $H$ is a non-abelian special 2-group which does not enjoy the Stone-Von Neumann property (Corollary 13).

\section{REFERENCES}

[BR97] J. Buhler, Z. Reichstein, On the essential dimension of a finite group, Compositio Math. 106 (1997), no. 2, 159-179.

[GH07] Sh. Gurevich, R. Hadani, The geometric Weil representation, Selecta Math. (N.S.) 13 (2007), no. 3, 465-481.

[H40] P. Hall, The classification of prime-power groups, J. Reine Angew. Math. 182 (1940), $130-141$.

[HH56] P. Hall, G. Higman, On the p-length of p-soluble groups and reduction theorem for Burnside's problem Proc. of London Math. Soc. (3), no. 6 (1956), 1-42.

[JNO90] R. James, M. F. Newman, E. A. O'Brien, The groups of order 128, J. Algebra 129 (1990), no. 1, 136-158.

[JLY02] C. U. Jensen, A. Ledet, N. Yui, Generic Polynomials: Constructive Aspects of The Inverse Galois Problem, Cambridge University Press, 2002. 
[KM07] N. A. Karpenko, A. S. Merkurjev, Essential dimension of finite p-groups, Inventiones Math., 172, no. 3 (2008), 491-508.

[MR09] A. Meyer, Z. Reichstein, Some consequences of the Karpenko-Merkurjev theorem, Documenta Math., Extra Volume dedicated to Andrei A. Suslin's Sixtieth Birthday (2010), 445-457.

[R04] J. Rosenberg, A selective history of the Stone-von Neumann theorem, Operator algebras, quantization, and noncommutative geometry, 331-353, Contemp. Math. 365, Amer. Math. Soc., Providence, RI, 2004.

[TZ00] P. H. Tiep, A. E. Zalesskii, Some aspects of finite linear groups: a survey, Algebra, 12, J. Math. Sci. (New York) 100 (2000), no. 1, 1893-1914.

[W03] S. H. Weintraub, Representation theory of finite groups: algebra and arithmetic, Graduate Studies in Mathematics, 59, American Mathematical Society, Providence, RI, 2003.

Department of Mathematics, University of British Columbia, Vancouver, BC V6T 1Z2, CANADA

SCERNELE@MATH.UBC.CA, MASOUd@MATH.UBC.CA, REICHST@MATH.UBC.CA 